Research Article

\title{
Dynamic Inversion Analysis of Structural Layer Modulus of Semirigid Base Pavement considering the Influence of Temperature and Humidity
}

\author{
Bei Zhang, Xu Zhang, Yanhui Zhong $\mathbb{D}$, Xiaolong Li $\mathbb{D}$, Meimei Hao, and Jinbo Liu \\ College of Water Conservancy and Engineering, Zhengzhou University, Zhengzhou 450001, China \\ Correspondence should be addressed to Yanhui Zhong; zhong_yanhui@163.com and \\ Xiaolong Li; wennuandeshang@hotmail.com
}

Received 29 July 2020; Revised 11 September 2020; Accepted 27 September 2020; Published 29 October 2020

Academic Editor: Grzegorz Golewski

Copyright (c) 2020 Bei Zhang et al. This is an open access article distributed under the Creative Commons Attribution License, which permits unrestricted use, distribution, and reproduction in any medium, provided the original work is properly cited.

This paper is aimed to solve the overlearning problem of the neural network algorithm used to calculate the asphalt concrete pavement structural modulus in reverse. The firefly algorithm was adapted to optimize the selection of support vector machine (SVM) parameters. Based on the optimized SVM model, a new method for dynamic inversion of the semirigid base asphalt concrete pavement structural layer modulus was presented. The results show that the absolute value of relative error of each layer modulus is not more than $3.73 \%$ by using the proposed method. Then, the influences of temperature and humidity on the inversion modulus of semirigid base asphalt concrete pavement in the seasonal frozen area were analyzed, and the correction formula of the inversion modulus was established. The paper is of practical significance for improving the safety performance of semirigid base pavement in the seasonal frozen area in China.

\section{Introduction}

In recent years, China's highway construction has developed rapidly in terms of construction scale and quantity. The highway has reached a total mileage of 5 million kilometers by 2019 , including $143,000 \mathrm{~km}$ of expressways. The semirigid base asphalt concrete pavement is most widely used in expressways [1]. In seasonal frozen areas such as Northeast China, Inner Mongolia, Qinghai, Gansu, Ningxia, and Tibet, the asphalt concrete pavement structure bears the frostthawing damage under low temperature and damp environment, and its bearing capacity and stability of material will be greatly affected. These factors result in pavement cracks, cavities, subsidence, rutting, and other diseases, which seriously affect the traffic operation and normal use of expressways and even threaten the development of society and economy [2]. At present, in the design methods of asphalt pavement in the world, static load or approximate equivalent static load is generally used, but the static load mode cannot reflect the actual stress state of the pavement, so the influence of dynamic load on the design of the pavement structure has become the focus of research. Golewski studies the design of the concrete structure under dynamic load and puts forward the reference criteria for the design, construction, and maintenance of the dynamic loading structure, which provides a reference method for the design of pavement concrete structure $[3,4]$.

The bearing capacity of semirigid base asphalt concrete pavement is important to evaluate pavement performance. Falling weight deflectometer (FWD) is widely used as a kind of nondestructive testing equipment for the asphalt concrete pavement structure. Its principle is to carry out inversion analysis on the modulus of the asphalt concrete pavement structural layer according to the deflection value measured by FWD under impact load [4]. Scrivener et al. first proposed the inverse modulus calculation method of FWD deflection basin by solving the Burmister double layer system and compiled the inversion nomograph [5]. However, the regression analysis method cannot be used to process largescale FWD-measured data with poor inversion accuracy and 
versatility. Bush developed BISDEF and CHEVDEF software for inversion of the pavement structural layer modulus by using the iterative method [6]. Based on the system identification, Wang used this method to develop a software SIDMOD for inversion of the pavement structural layer modulus [7]. Nevertheless, the inversion results obtained by the iterative method are greatly affected by initial value and iterative method, and the iterative method converges slowly. Based on the database search method, Utahj et al. developed the MODULUS software for inversion of the pavement structural layer modulus [8]. Nonetheless, the database search method takes a long time to calculate and needs to select a more strict range of modulus. Meier et al. used the BP neural network to inverse the modulus and developed ANOVA software for inversion of pavement structural layer modulus [9]. There are some problems in using the neural network method such as oscillation and divergence out of improper initial value. Fwa et al. studied the inverse calculation of modulus by adopting the genetic algorithm and developed the inverse software NUS-GABACK [10]. Due to the slow calculation speed, of the genetic algorithm, NUSGABACK software cannot realize the large-scale inverse calculation needed in practical engineering. The above studies are all static inversion methods. Wang developed a dynamic inversion program integrated with the artificial neural network and genetic algorithm (ANN-GA) to calculate the modulus of the structural layer. According to the asphalt concrete pavement deflection measured by FWD, the service condition of asphalt pavement can be evaluated [11]. You et al. developed a dynamic inversion model based on ANN to predict the interlayer contact conditions and structural layer modulus of the pavement structure in view of the asphalt concrete pavement interlayer contact conditions [12]. Considering the influence of FWD dynamic load characteristics, viscoelastic material parameters, and nonlinear material parameters, $\mathrm{Li}$ et al. developed a dynamic inversion program based on ANN in combination with the genetic algorithm [13]. However, the neural network may cause the problem of overlearning.

Asphalt concrete pavement material is a kind of viscoelastic material whose strength and stability are easily affected by temperature changes, so the bearing capacity of the asphalt concrete pavement structure will change with temperature. Subgrade is a linear structure built in the natural environment, and its structural performance will change with the freeze-thaw cycle in the seasonal frozen area. The specific performance is that the change of seasonal climate causes the moisture in the subgrade to freeze-thaw. This affects the structural performance of the subgrade and ultimately leads to the destruction of the asphalt concrete pavement structure. Therefore, the influence of temperature and humidity should be considered when calculating the modulus of the pavement structure layer. Scholars have carried out a lot of research on the relationship between temperature and humidity in seasonally frozen areas and the modulus of pavement material. Chamberlain et al. found that the pore ratio of fine-grained soil changes with freezethaw cycle and modulus, and the permeability increases with the continuous freeze-thaw cycle [14]. Observing the change law of soil material properties after the first freeze-thaw change, Simonsen et al. found that the modulus value of subgrade decreased after the first freeze-thaw [15]. Taking remolded soil as the research object, Mao studied the change law of soil modulus of resilience with water content and freeze-thaw cycles. It was found that water content in subgrade is the most important factor affecting the modulus of resilience, and the freeze-thaw cycle is a secondary one under the conditions of optimum water content and degree of compaction [16]. Chen used the self-developed multifunctional subgrade freeze-thaw cycle test device to paper the repeated freeze-thaw characteristics of subgrade soil in a seasonal frozen area under different water compensating state. Analyzing the influence of initial degree of compaction, freeze-thaw cycle times, and water content on modulus of resilience, he revealed the change law of modulus of resilience and gave the modulus reduction coefficient of subgrade in a seasonal frozen area [17]. Wang used the selfdeveloped remote temperature and humidity acquisition system for subgrade in a seasonal frozen area to monitor the temperature and humidity status of subgrade, revealing the temperature and humidity change law of pavement structure in the seasonal frozen area. Meanwhile, he also analyzed the influence of water content, freeze-thaw cycle times, and degree of compaction on subgrade strength in a seasonal frozen area through the indoor freeze-thaw cycle test [18]. To sum up, although there are many research studies on the asphalt concrete pavement in the seasonal frozen area, most of them are about the influence of environmental factors on pavement materials and subgrade physical characteristics in the frozen soil area. There is no relevant research on the influence of temperature and humidity in the seasonal frozen area on the inversion modulus of the semirigid base pavement structure layer.

Therefore, this paper introduces the SVM method, optimizes the inversion of the asphalt concrete pavement structural layer modulus by the firefly algorithm considering the effect of temperature and humidity, and analyzes the influence of temperature and humidity on the inversion modulus of semirigid base asphalt pavement in the seasonal frozen area. The research results have guiding significance to ensure the safety performance of the asphalt concrete pavement structure in the seasonal frozen area and extend its service cycle.

\section{Dynamic Response Analysis of Semirigid Base Pavement}

\subsection{Dynamic Finite Element Analysis Model of Pavement Structure}

2.1.1. Basic Assumption. In this paper, the displacement of the elastic layer system is analyzed by assuming that each layer satisfies the following conditions:

(1) Each structural layer is homogeneous and isotropic, which satisfies the deformation law of linear elastic body

(2) The contact surface of each structural layer is continuous 
(3) The subgrade extends infinitely in horizontal and longitudinal directions

2.1.2. Semirigid Base Pavement Structure Model. This paper takes FWD load as the research object and selects the depth direction $6 \mathrm{~m}$ and radial direction $6 \mathrm{~m}$ as calculation elements. According to the theory of pavement structure mechanics, the displacement, stress, and strain of the axisymmetric structure have nothing to do with the value of $\theta$, but only with the value of $R$ and $Z$. Therefore, the rectangular ring element is used as the research object of the axisymmetric structure, and only the section on the coordinate $R$ and $z$ plane needs to be studied. The finite element model is shown in Figure 1.

2.2. Calculation of Pavement Dynamic Response. According to Hamilton's variation principle, the dynamic balance equation considering damping effect is [19]

$$
\begin{gathered}
{[M]\{\ddot{\delta}\}+[C]\{\dot{\delta}\}+[K]\{\delta\}=\{P\},} \\
{[C]=\alpha[M]+\beta[M],}
\end{gathered}
$$

where $[M]$ is the global mass matrix; $\{\ddot{\delta}\}$ is the global acceleration vector; $[C]$ is the global damping matrix; $\{\dot{\delta}\}$ is the global velocity vector; $[K]$ is the global stiffness matrix ; $\{\delta\}$ is the global displacement vector; $\{P\}$ is the equivalent node load vector; $\alpha$ and $\beta$ are the damping coefficients, $\alpha=\xi \omega_{1}$ and $\beta=\xi / \omega_{1} ; \omega_{1}$ is the structural fundamental frequency; $\xi$ is the mode damping ratio.

The calculation range of the model, boundary treatment, and characteristics of the rectangular ring element are known from $[20,21]$.

In this paper, the Wilson- $\theta$ direct integration method $[20,21]$ is selected to calculate the dynamic balance equation, and $\theta=1.4$ is taken in the calculation. The specific steps are as follows.

2.2.1. Initial Calculation. Calculate the following constants:

$$
\begin{aligned}
\tau & =\theta \Delta t(\theta=1.4), \\
b_{0} & =\frac{6}{\tau^{2}}, b_{1}=\frac{3}{\tau}, b_{2}=2 b_{1}, b_{3}=\frac{\tau}{2}, b_{4}=\frac{b_{0}}{\theta}, \\
b_{5} & =\frac{b_{2}}{\theta}, b_{6}=1-\frac{3}{\theta}, b_{7}=\frac{\Delta t}{2}, b_{8}=\frac{\Delta t^{2}}{6} .
\end{aligned}
$$

The effective stiffness matrix is established:

$$
[\bar{K}]=[K]+b_{1}[C]+b_{0}[M] .
$$

The effective stiffness matrix is decomposed:

$$
[\bar{K}]=[L][D][L]^{\mathrm{T}} .
$$

2.2.2. Calculation of Each Time Step. Calculate the effective load vector:

$$
\begin{aligned}
\left\{\bar{P}\left(t_{1}+\tau\right)\right\}= & \left\{P\left(t_{1}+\tau\right)\right\}+[M]\left(2\left\{\ddot{\delta}\left(t_{1}\right)\right\}+b_{2}\left\{\dot{\delta}\left(t_{1}\right)\right\}\right. \\
& \left.+b_{0}\left\{\delta\left(t_{1}\right)\right\}\right)+[C]\left(b_{3}\left\{\ddot{\delta}\left(t_{1}\right)\right\}\right. \\
& \left.+2\left\{\dot{\delta}\left(t_{1}\right)\right\}+b_{1}\left\{\delta\left(t_{1}\right)\right\}\right) .
\end{aligned}
$$

The equation of motion is solved by

$$
\left\{\delta\left(t_{1}+\tau\right)\right\}=[\bar{K}]^{-1}\left\{\bar{P}\left(t_{1}+\tau\right)\right\} .
$$

Calculate acceleration, velocity, and displacement of $t_{1}+$ $\Delta t$ time:

$$
\begin{aligned}
\ddot{\delta}\left(t_{1}+\Delta t\right)= & b_{4}\left[\delta\left(t_{1}+\Delta t\right)-\delta\left(t_{1}\right)\right] \\
& +b_{5} \dot{\delta}\left(t_{1}\right)+b_{6} \ddot{\delta}\left(t_{1}\right) \\
\dot{\delta}\left(t_{1}+\Delta t\right)= & \dot{\delta}\left(t_{1}\right)+b_{7}\left[\ddot{\delta}\left(t_{1}\right)+\ddot{\delta}\left(t_{1}+\Delta t\right)\right], \\
\delta\left(t_{1}+\Delta t\right)= & \delta\left(t_{1}\right)+\dot{\delta}\left(t_{1}\right) \Delta t+b_{8}\left[\ddot{\delta}\left(t_{1}+\Delta t\right)+2 \ddot{\delta}\left(t_{1}\right)\right] .
\end{aligned}
$$

Based on the above solution method, the dynamic response calculation program of the semirigid base pavement structure is compiled in this paper.

\subsection{Example Analysis}

(1) In a three-layer semirigid base pavement system, the elastic modulus of each layer is, respectively, $1200 \mathrm{MPa}, 1400 \mathrm{Mpa}$, and $60 \mathrm{MPa}$, the thickness $18 \mathrm{~cm}, 32 \mathrm{~cm}$, and $550 \mathrm{~cm}$, the Poisson's ratio 0.35 , 0.30 , and 0.40 , and the density $2400 \mathrm{~kg} / \mathrm{m}^{3}, 2300 \mathrm{~kg} /$ $\mathrm{m}^{3}$, and $1800 \mathrm{~kg} / \mathrm{m}^{3}$. In this paper, Dynatest 8000 FWD is used as the applied load. In order to simplify the calculation process, the load application curve of FWD is simplified to a sine semiperiodic function with the peak value of $721 \mathrm{kpa}$, the action time of $32 \mathrm{~ms}$, and the loading radius of $0.15 \mathrm{~m}$. The load time history curve and transducer distribution position of the simplified FWD are shown in Figure 2 and Table 1.

The calculated deflection results in this paper are basically consistent with the measured deflection basin data at different measuring points with small error and high accuracy from Figure 5, which can be applied to actual asphalt concrete pavement engineering.

Eight-node linear hexahedron element (C3D8R) is selected as the calculation element of the finite element model. The boundary conditions are set as follows: there is no $X$-direction displacement in the left-right direction and no $Y$-direction displacement in the front-back direction. All nodes on the bottom are completely fixed, and the layers are completely continuous. The finite element model is shown in Figure 3.

It can be seen from Figure 4 that the calculation results at different times obtained by applying the 


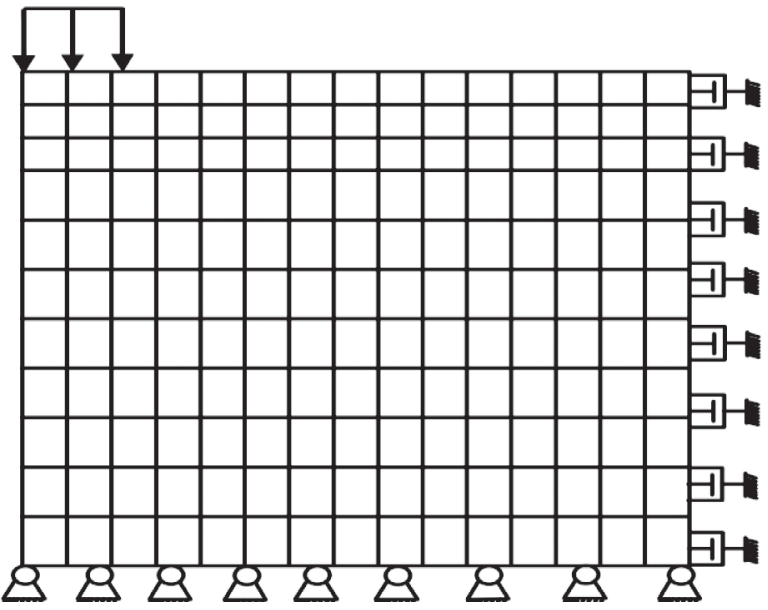

FIGURE 1: Finite element model of the pavement structure.

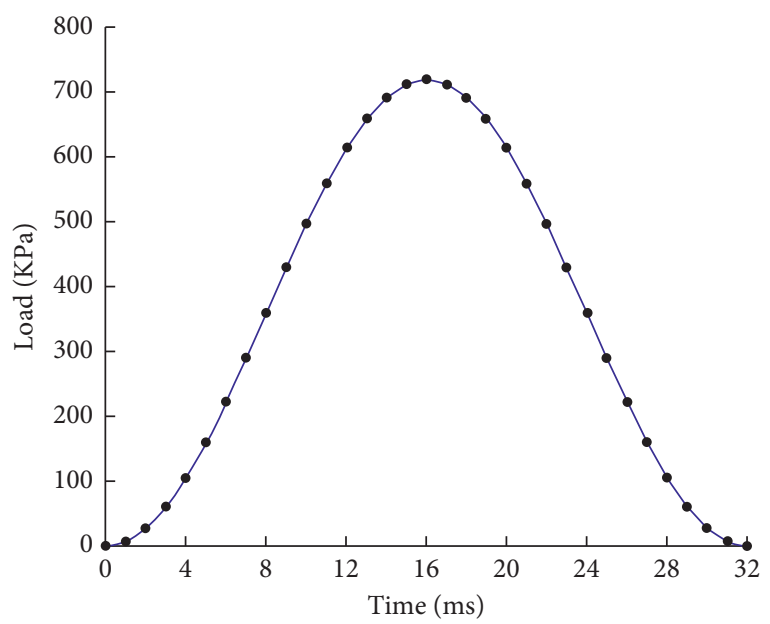

FIGURE 2: FWD load time history curve.

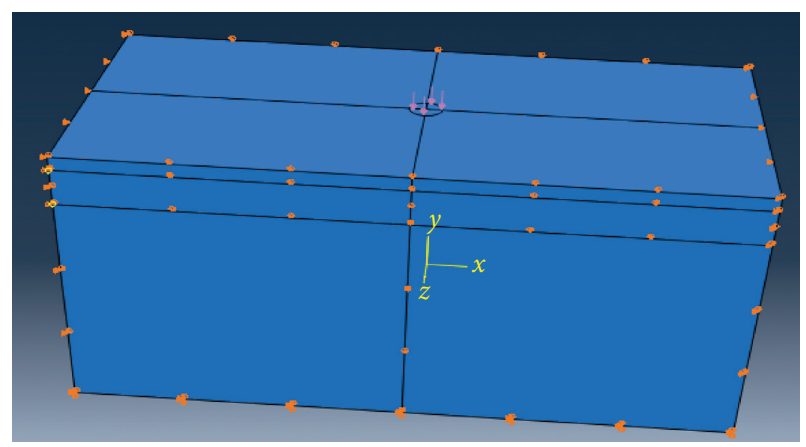

FIGURE 3: Finite element model.

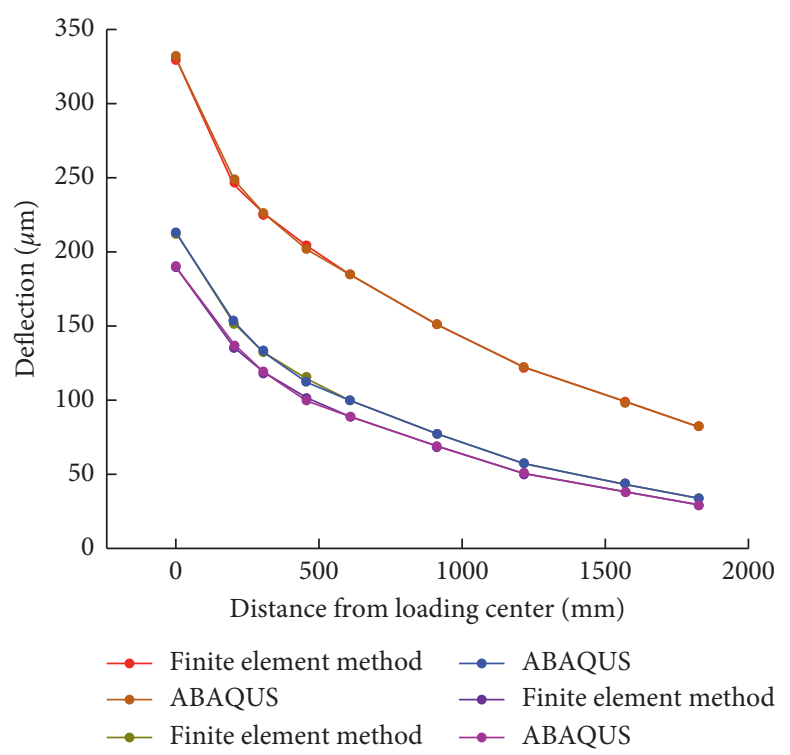

Figure 4: Comparison of pavement deflection calculation results at different time.

TABle 1: Dynatest8000 FWD transducer layout.

\begin{tabular}{lccccccccc}
\hline Transducer number & C1 & C2 & C3 & C4 & C5 & C6 & C7 & C8 & C9 \\
\hline Distance from loading center $(\mathrm{mm})$ & 0 & 204 & 305 & 457 & 610 & 914 & 1219 & 1574 & 1829 \\
\hline
\end{tabular}

method in this paper are basically consistent with those of ABAQUS, which proves that the dynamic response calculation program has high accuracy.

(2) In order to further verify the accuracy of the program, based on FWD-measured deflection basin data and the position of the measuring points in [22], the calculation program of this paper is used to calculate the corresponding data. The calculated results are compared with the measured data, as shown in Figure 5.

\section{Modulus Inversion of Asphalt Concrete Pavement Structural Layer Based on SVM}

3.1. Modulus Inversion of Asphalt Concrete Pavement Structural Layer. This paper introduces the SVM model in the modulus inversion of the asphalt concrete pavement structure layer and uses the SVM model instead of the numerical model to carry out the inversion of the modulus, considering the disadvantages of the previous inversion methods of pavement modulus. Based on the structural risk minimization criterion, SVM can balance the relationship between training error and complexity of learning machine. It can improve inversion efficiency in the application of engineering practice. SVM can solve the "overlearning" problem faced by the neural network well and make the calculation results have better generalization ability. It has been widely used in the fields of automatic control, pattern recognition, and rock mechanic parameter inversion [23].

The inversion process of the pavement structural modulus using the SVM model is as follows [24]. 
The standard $\operatorname{SVM}$ function $\operatorname{SVM}\left(x_{1}, x_{2}, \ldots, x_{m}\right)$ is used to reflect the nonlinear relationship between deflection and parameters to be inversed:

$$
\begin{aligned}
& \operatorname{SVM}(X): R^{m} \longrightarrow R, \\
& y=\operatorname{SVM}(X), \\
& X=\left(x_{1}, x_{2}, \ldots, x_{m}\right),
\end{aligned}
$$

where $X=\left(x_{1}, x_{2}, \ldots, x_{m}\right)$ is the parameter of the pavement structural layer to be inverted and $Y$ is the deflection value of a measuring point measured in the field.

The number of $\operatorname{SVM}\left(x_{1}, x_{2}, \ldots, x_{m}\right)$ is the same as the number of deflection transducer, and $n$ SVM models correspond to $n$ deflection values. The SVM model $y(X)$ which reflects the mapping relationship between structural layer modulus and pavement deflection is established by enough learning samples:

$$
y(X)=\sum_{i=1}^{l}\left(\alpha_{i}-\alpha_{i}^{\prime}\right) k\left(x, x_{i}\right)+b,
$$

where $y(X)$ is a set of displacements of a line corresponding to parameter $X=\left(x_{1}, x_{2}, \ldots, x_{m}\right) ; l$ is the number of learning samples; $k\left(x, x_{i}\right)$ is the kernel function; $\alpha, \alpha^{*}$ is the Lagrange multiplier of the SVM algorithm.

From the SVM model, it can be seen that the parameter $\alpha, \alpha^{*}$ and the offset $b$ are important parameters for creating the SVM model, and their values can be obtained by solving the constrained quadratic programming problem:

$$
\begin{aligned}
\max W\left(\alpha, \alpha^{*}\right)= & -\frac{1}{2} \sum_{i, j=1}^{l}\left(\alpha_{i}-\alpha_{i}^{*}\right)\left(\alpha_{j}-\alpha_{j}^{*}\right) k\left(x_{i}, x_{j}\right) \\
& +\sum_{i=1}^{l} y_{i}\left(\alpha_{i}-\alpha_{i}^{*}\right)-\varepsilon \sum_{i=1}^{l}\left(\alpha_{i}+\alpha_{i}^{*}\right),
\end{aligned}
$$

$$
\text { s.t. } \sum_{i=1}^{n}\left(a_{i}-a_{i}^{*}\right)=0,0 \leq a_{i}, a_{i}^{*} \leq C, \quad i=1, \ldots, n .
$$

An efficient optimization algorithm is used to solve the parameters $\alpha, \alpha^{*}$ and the offset $b$ to establish the standard SVM model. The optimal solution is constantly searched in the range of modulus. When the error between the deflection value calculated by the SVM model and the measured deflection reaches the minimum, a set of modulus parameters corresponding to the calculated deflection can be determined as the real modulus of the asphalt concrete pavement structure layer. The inversion process is completed.

\subsection{Basic Steps of SVM Inversion Optimization Based on} Firefly Algorithm. The selection and optimization of model parameters have a great influence on the establishment of SVM model. The reasonable model parameters are one of the important factors to ensure the accuracy and reliability of the inversion results of the SVM model. The SVM model has

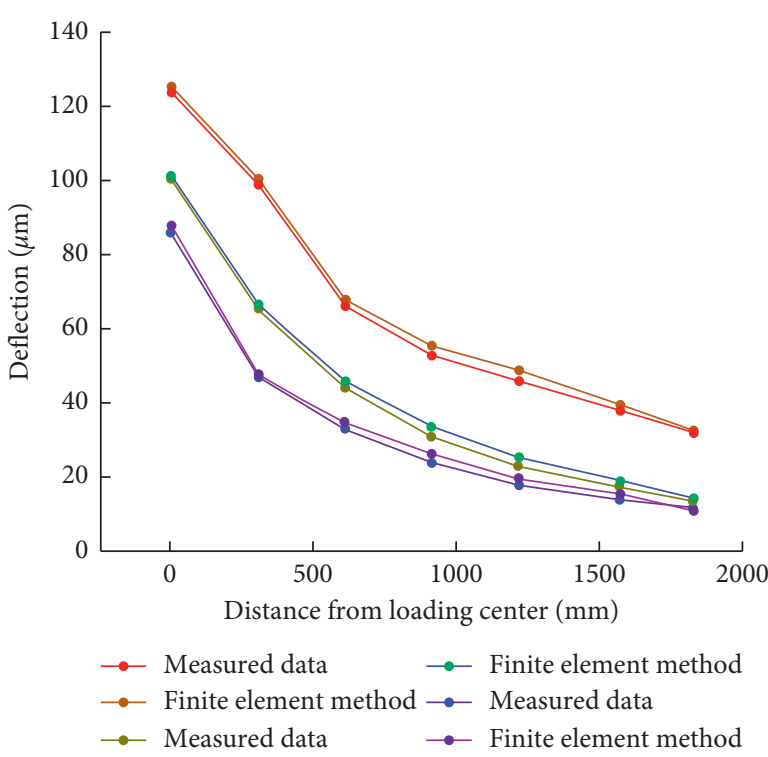

Figure 5: Comparison of deflection results at different measuring points.

two parameters, the basic parameter of the model (such as penalty factor $C$ and insensitive loss parameter $\varepsilon$ ) and the parameter related to kernel function (such as the kernel width $\sigma$ in radial basis function, the constant $c$ in polynomial kernel function, and the exponential function $d$ ). Previous methods of parameter selection mainly rely on subjective experience and grid search, so it is difficult to find the best parameters [25,26]. In recent years, scholars use the heuristic intelligent search algorithm with global optimization ability to select parameters of SVM and get good results. Therefore, the firefly algorithm is used to obtain the optimal parameters of the SVM model in this paper.

3.2.1. Introduction of Firefly Algorithm. Suppose $n$ fireflies are randomly and evenly distributed in the solution space and each firefly is given a unique initial brightness value. The function value of the firefly's position will determine the brightness of the firefly. The relationship between the position and the brightness of the firefly is the better the position, the higher the brightness. Each firefly has its own dynamic decision domain $r_{d}^{i}$, in which the firefly moves towards the brighter firefly, forming a domain set. Then, the position, brightness, and decision domain of the firefly are updated [27]. The main phases of the firefly algorithm are as follows:

(1) The updating phrase of the dynamic decision domain:

$$
r_{d}^{i}(t+1)=\min \left\{r_{s}, \max \left\{0, r_{d}^{i}(t)+\beta\left(n_{t}-\left|N_{t}(t)\right|\right)\right\},\right.
$$

where $r_{d}^{i}(t+1)$ is the $t+1$ generation decision range of the $t$ generation firefly $i ; r_{s}$ is the perception range of the firefly; $n_{t}$ is the domain threshold which can control the number of fireflies; $\beta$ is the constant 
related to the decision domain; $N_{t}(t)$ is the number of fireflies within the radius of $r_{d}^{i}$.

(2) The updating phrase of position:

In this phase, each firefly first selects the firefly whose brightness is higher than itself within the range of its decision domain to form the domain set. The formula is

$N_{t}(t)=\left\{j:\left\|x_{j}(t)-x_{i}(t)\right\|<r_{d}^{i}, l_{i}(t)<l_{j}(t)\right\}$,

where $\left\|x_{j}(t)-x_{i}(t)\right\|$ is the Euclidean distance between two fireflies; $x_{j}(t)$ is the firefly position; $l_{i}(t)$ is the fluorescein value of the $t$ iteration.

The formula of the moving probability is as follows:

$$
p_{i j}(t)=\frac{l_{j}(t)-l_{t}(t)}{\sum_{k \in N_{t}(t)} l_{k}(t)-l_{i}(t)},
$$

where $p_{i j}$ is the probability of firefly $i$ moving to firefly $j$. After the composition of the domain set, the position is updated according to the following formula:

$$
x_{i}(t+1)=x_{i}(t)+s\left[\frac{x_{j}(t)-x_{i}(t)}{\left\|x_{j}(t)-x_{i}(t)\right\|}\right] .
$$

(3) The updating phrase of updating fluorescein value:

$$
l_{i}(t)=(1-p) l_{i}(t-1)+\gamma J\left(x_{i}(t)\right)
$$

where $l_{i}(t)$ represents the fluorescein value of the firefly $i$ of the $t$ generation; $p$ is the vanishing rate; $\gamma$ is the parameter to measure the fitness function value; $J\left(x_{i}(t)\right)$ is the fitness function value.

The main steps of the firefly algorithm are as follows:

(1) Random initialization of firefliesi $(i=1,2, \ldots, n)$

(2) Calculating the function value $J\left(x_{i}(t)\right)$ corresponding to the position $x_{i}(t)$ of the firefly $i$ in the $t$ generation and the fluorescein value $l_{i}(t)$ of the current firefly by formula (20), so as to update the fluorescein value of the firefly

(3) Each firefly looks for the individuals with higher fluorescein value in its dynamic decision domain radius $r_{d}^{i}$ and forms the domain set $N_{t}(t)$ together with the higher fluorescein value

(4) Getting the moving probability $p_{i j}(t)$ of firefly $i$ into the domain set by formula (18)

(5) Obtaining the moving position of firefly by formula (17)

(6) Updating the radius of dynamic decision domain by formula (16)

3.2.2. Optimization of SVM Model Parameters Based on Firefly Algorithm. This paper adopts the firefly algorithm to optimize the SVM model and then solves the problem of "overlearning" and "underlearning" when the SVM model is not the optimal parameter.
The specific steps of the firefly algorithm to optimize SVM model parameters are as follows:

(1) Giving initial values to the parameters of each firefly, and setting the initial position of the firefly with the range of initial position parameters $\left(C_{\min } \sim C_{\max }\right.$, $\left.\sigma_{\min } \sim \sigma_{\max }\right)$ and the maximum number of iterations;

(2) Calculating brightness value as brightness update;

(3) Obtaining the domain set of each firefly and randomly selecting the firefly $i$ in the set as its moving direction;

(4) Calculating the training value of SVM after position update. If the training value of the updated SVM is better than the previous one, the position will be replaced, otherwise it will not change;

(5) Updating the dynamic decision domain;

(6) Calculating the above process in cycle. If the search times reach its maximum, the cycle process will be interrupted. The SVM will be trained according to the optimal solution in the process until the end condition is satisfied.

\subsection{Example Analysis}

(1) The thickness of each layer of semirigid base asphalt concrete pavement is $18 \mathrm{~mm}, 32 \mathrm{~mm}$, and $\infty$, respectively. Poisson's ratio of each layer is $0.35,0.30$, and 0.40 . The number of transducers is $d_{1} \sim d_{7}$. The position of each transducer is $0.0 \mathrm{~mm}, 304.8 \mathrm{~mm}$, $609.6 \mathrm{~mm}, 914.4 \mathrm{~mm}, 1219.2 \mathrm{~mm}, 1524.0 \mathrm{~mm}$, and $1828.8 \mathrm{~mm}$, respectively.

In this paper, seven groups of different modulus parameters are set up to test the correctness of the inversion results. The corresponding deflection values of each parameter combination are shown in Table 2. The range of modulus parameters of each layer is $1000-4000 \mathrm{MPa}$ for surface course, 400-3000 MPa for base course, and 30-150 MPa for subgrade. The modulus parameters of each layer are substituted into the dynamic response calculation program to obtain the corresponding pavement deflection. These data form a sample pair, and thus 12 test samples and 100 learning samples are obtained.

It can be seen from Figure 6 that the modulus of asphalt concrete pavement surface course, base layer, and subgrade obtained by this method is between the regression model algorithm and the BP model algorithm. Furthermore, the inversion results have good stability by this method. The three methods are basically consistent with each other, which proves that the method in this paper has high accuracy and completely meets the practical engineering calculation requirements.

This paper adopts standard SVM in which the loss function is an insensitive loss function and the kernel function is a radial basis kernel function (RBF) [28]. Through the training of learning samples and test 
TABLE 2: Seven sets of deflection basin data.

\begin{tabular}{|c|c|c|c|c|c|c|c|c|c|c|}
\hline \multirow{2}{*}{ Number } & \multirow{2}{*}{ Surface course $(\mathrm{MPa})$} & \multirow{2}{*}{ Base course (MPa) } & \multirow{2}{*}{ Subgrade (MPa) } & \multicolumn{7}{|c|}{ Theoretical deflection $(\mu \mathrm{m})$} \\
\hline & & & & $\mathrm{d} 1$ & $\mathrm{~d} 2$ & d3 & $\mathrm{d} 4$ & d5 & d6 & $\mathrm{d} 7$ \\
\hline 1 & 1200 & 1400 & 60 & 329.63 & 225.14 & 184.91 & 150.78 & 121.96 & 99.19 & 82.42 \\
\hline 2 & 2330 & 1700 & 110 & 293.24 & 199.33 & 162.29 & 132.15 & 107.16 & 87.43 & 72.74 \\
\hline 3 & 1550 & 2200 & 140 & 288.98 & 190.19 & 154.85 & 125.80 & 101.77 & 82.82 & 69.02 \\
\hline 4 & 3600 & 1300 & 35 & 318.47 & 232.88 & 192.81 & 159.02 & 130.09 & 106.65 & 88.16 \\
\hline 5 & 2350 & 800 & 50 & 331.95 & 234.87 & 190.54 & 155.04 & 125.74 & 102.62 & 84.88 \\
\hline 6 & 3200 & 1400 & 90 & 294.92 & 204.57 & 166.33 & 135.55 & 110.05 & 89.91 & 74.76 \\
\hline 7 & 3800 & 1100 & 140 & 286.91 & 195.02 & 155.67 & 125.50 & 101.24 & 82.55 & 68.88 \\
\hline
\end{tabular}

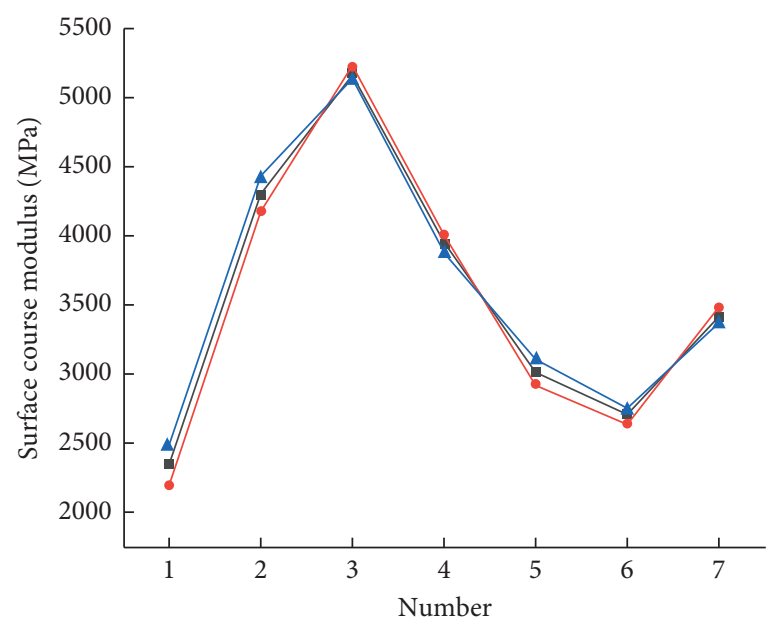

- SVM model algorithm

$\rightarrow$ Regression model algorithm

$\neg$ BP model algorithm

(a)

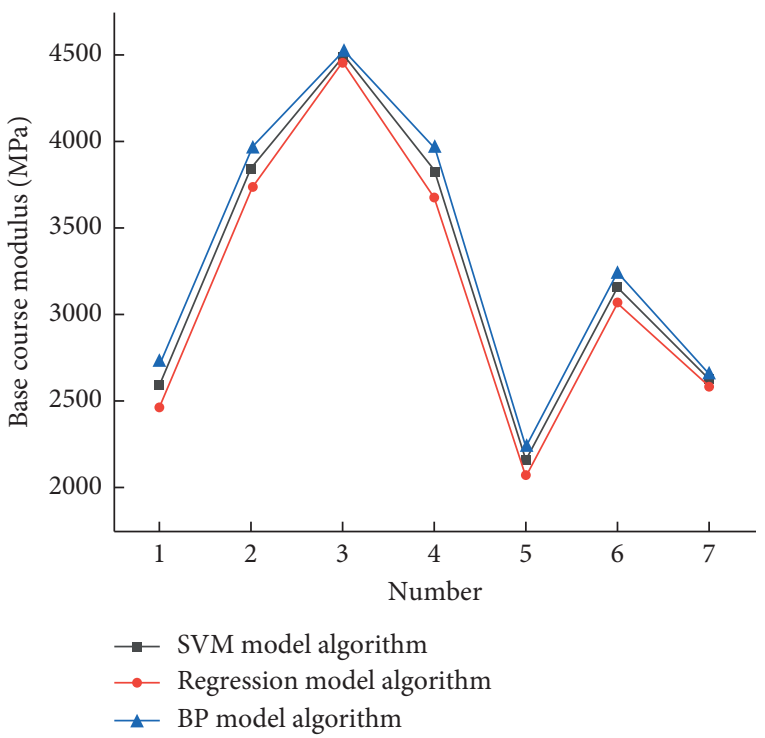

(b)

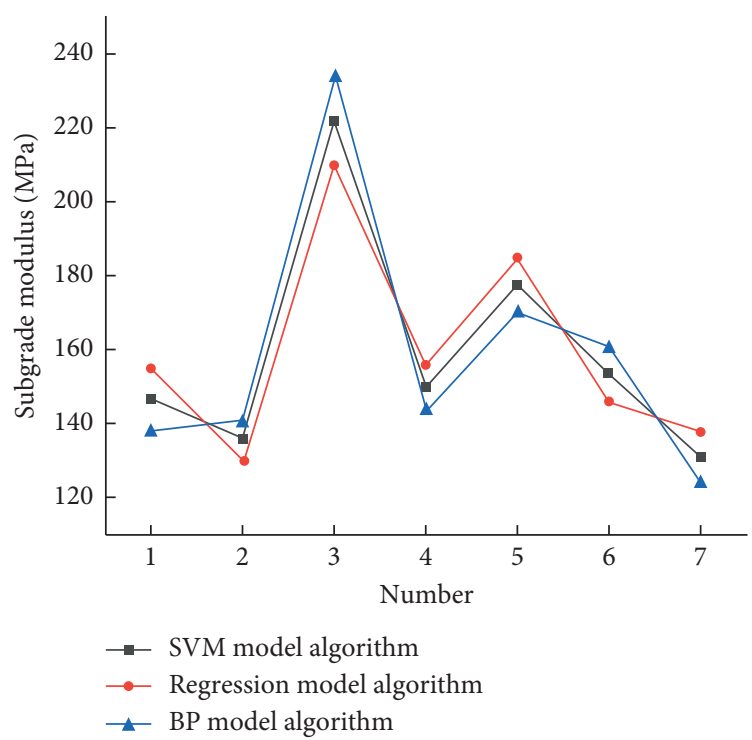

(c)

Figure 6: Comparison of inversion results of different inversion methods. 
samples, the optimal model parameters and the offset $b$ values of SVM corresponding to each deflection transducer are obtained, as shown in Table 3.

After obtaining the optimal SVM model, the SVM model instead of the numerical model combines with the firefly algorithm to carry out the inversion. The inversion results are shown in Table 4.

The results reveal that the average absolute value of relative error of surface course modulus inversion results is $0.97 \%$, and the maximum value is of $2.16 \%$. The average absolute value of relative error absolute value of the base course modulus inversion result is $0.82 \%$, and the maximum value is of $2.31 \%$. The average absolute value of relative error absolute value of the subgrade structure layer modulus prediction result is $1.91 \%$, and the maximum value is of $3.73 \%$. Based on the results, the SVM model has high inversion efficiency and accuracy, which can fully meet the practical engineering demands.

(2) In order to further verify the accuracy of the inversion program, the inversion method proposed in this paper is used to carry out the modulus inversion of each structural layer of pavement with the measured deflection data [22]. The results are compared with those of the two methods in [22]. The results are presented in Table 5.

\section{Correction of Modulus Inversion in Seasonal Frozen Area}

4.1. Temperature Correction of Inversion Surface Modulus in Seasonal Frozen Area. For asphalt concrete pavement, the asphalt mixture is a kind of temperature sensitive material. Its strength, stiffness, and deformation resistance will change with temperature. When the asphalt concrete pavement temperature increases, the strength and stiffness of the asphalt mixture will decrease, causing the asphalt concrete pavement to easily undergo high-temperature shear failure under the vehicular load. When the asphalt concrete pavement temperature decreases, the deformation capacity of the asphalt mixture decreases, causing the asphalt concrete pavement to easily undergo low-temperature shrinkage cracking. Pavement modulus which can reflect the strength and stiffness of pavement is the main parameter of pavement structure design. Therefore, the analysis of the relationship between asphalt concrete pavement surface modulus and temperature is of great significance to the evaluation of pavement structure bearing capacity in the seasonal frozen area.

In this paper, the temperature field model of Zhang is selected as the research object [29]. The model is as follows.

Pavement temperature along the depth direction:

$$
\begin{aligned}
T_{H}= & T_{60}+\left(T_{s}-T_{60}\right) \times\left(a \times H^{3}+b \times H^{2}+c \times H+d\right) \\
& \times \exp (e H),
\end{aligned}
$$

where $H$ is the distance between a certain point within the asphalt concrete pavement and the surface; $T_{H}$ is the temperature at the depth of $h ; T_{60}$ is the temperature at $60 \mathrm{~cm}$ of subgrade; $T_{S}$ is the asphalt concrete pavement temperature or actual pavement temperature; $a \sim e$ is the undetermined regression coefficient.

According to the hot linear elastic layer system theory and in consideration of the structural characteristics and stress state of asphalt pavement, the following assumptions are set:

(1) Each structural layer of the asphalt concrete pavement is isotropic and homogeneous thermoelasticity material

(2) It is assumed that only the material parameters of the surface course are affected by temperature and the material parameters of the base course and subgrade are regarded as constant

(3) The structural layers are completely continuous

Based on the above hypothesis analysis, the following calculation examples are constructed for analysis: a threelayer semirigid base pavement system in the seasonal frozen area is selected, including $18 \mathrm{~cm}$ asphalt concrete surface course and $32 \mathrm{~cm}$ cement-stabilized macadam base and subgrade. Poisson's ratio of each layer in the asphalt concrete pavement is, respectively, $0.35,0.30$, and 0.40 and the density is $2400 \mathrm{~kg} / \mathrm{m}^{3}, 2300 \mathrm{~kg} / \mathrm{m}^{3}$, and $1800 \mathrm{~kg} / \mathrm{m}^{3}$.

The relationship between the inversion modulus of asphalt concrete pavement surface course and temperature can be obtained by embedding the temperature field prediction model into the inversion program. The calculation results are shown in Figure 7 and Table 6.

The inverse modulus of asphalt pavement has a good exponential relationship with the asphalt concrete pavement temperature from Figure 7. The inverse modulus of the surface course will decrease with the increase in temperature. The correlation formula of $E-T$ is obtained by regression analysis:

$$
E=2110 e^{-0.033 T} \text {. }
$$

The correlation coefficient $R^{2}$ between them is equal to 0.9896 .

The temperature correction coefficient $K$ is defined as the ratio of the modulus $E$ of asphalt pavement under $T$ temperature and the modulus $E_{S}$ under standard temperature $T_{S} . T_{S}$ is taken as $20^{\circ} \mathrm{C}$, that is,

$$
K=\frac{E}{E_{S}}=\frac{2110 e^{-0.033 T}}{2110 e^{-0.033 T_{S}}}=e^{-0.033(T-20)}
$$


TABle 3: Model training results.

\begin{tabular}{lccccccc}
\hline Position $(\mathrm{mm})$ & 0 & 304.8 & 609.6 & 914.4 & 1219.2 & 1524.0 & 1828.8 \\
\hline$C$ & 2000 & 1870 & 1968 & 1580 & 1856 & 1920 & 1740 \\
$\sigma$ & 2.35 & 2.36 & 2.36 & 2.37 & 2.37 & 2.38 & 2.39 \\
$b$ & 109.74 & 104.06 & 97.43 & 90.17 & 82.90 & 75.88 & 69.27 \\
\hline
\end{tabular}

TABLE 4: Modulus inversion results.

\begin{tabular}{|c|c|c|c|c|}
\hline Number & Structural layer & Structural layer modulus (MPa) & Inverse modulus (MPa) & Inversion relative error (\%) \\
\hline \multirow{3}{*}{1} & Surface course & 1200 & 1190 & -0.83 \\
\hline & Base course & 1400 & 1404 & 0.29 \\
\hline & Subgrade & 60 & 58 & -2.77 \\
\hline \multirow{3}{*}{2} & Surface course & 2330 & 2346 & 0.70 \\
\hline & Base course & 1700 & 1702 & 0.14 \\
\hline & Subgrade & 110 & 106 & -3.73 \\
\hline \multirow{3}{*}{3} & Surface course & 1550 & 1533 & -1.12 \\
\hline & Base course & 2200 & 2229 & 1.30 \\
\hline & Subgrade & 140 & 140 & -0.07 \\
\hline \multirow{3}{*}{4} & Surface course & 3600 & 3618 & 0.49 \\
\hline & Base course & 1300 & 1290 & -0.79 \\
\hline & Subgrade & 35 & 34 & -2.86 \\
\hline \multirow{3}{*}{5} & Surface course & 2350 & 2401 & 2.16 \\
\hline & Base course & 800 & 819 & 2.31 \\
\hline & Subgrade & 50 & 50 & 0.00 \\
\hline \multirow{3}{*}{6} & Surface course & 3200 & 3169 & -0.98 \\
\hline & Base course & 1400 & 1413 & 0.90 \\
\hline & Subgrade & 90 & 91 & 1.11 \\
\hline \multirow{3}{*}{7} & Surface course & 3800 & 3819 & 0.49 \\
\hline & Base course & 1100 & 1100 & -0.01 \\
\hline & Subgrade & 140 & 144 & 2.86 \\
\hline
\end{tabular}

TABLE 5: Comparison of inversion results of different inversion methods.

\begin{tabular}{|c|c|c|c|c|c|c|c|c|c|}
\hline \multirow[b]{2}{*}{ Number } & \multicolumn{3}{|c|}{ Surface course modulus (MPa) } & \multicolumn{3}{|c|}{ Base course modulus (MPa) } & \multicolumn{3}{|c|}{ Subgrade modulus (MPa) } \\
\hline & $\begin{array}{l}\text { SVM model } \\
\text { algorithm }\end{array}$ & $\begin{array}{c}\text { Regression } \\
\text { model } \\
\text { algorithm }\end{array}$ & $\begin{array}{l}\text { BP model } \\
\text { algorithm }\end{array}$ & $\begin{array}{l}\text { SVM model } \\
\text { algorithm }\end{array}$ & $\begin{array}{c}\text { Regression } \\
\text { model } \\
\text { algorithm }\end{array}$ & $\begin{array}{l}\text { BP model } \\
\text { algorithm }\end{array}$ & $\begin{array}{l}\text { SVM model } \\
\text { algorithm }\end{array}$ & $\begin{array}{c}\text { Regression } \\
\text { model } \\
\text { algorithm }\end{array}$ & $\begin{array}{l}\text { BP model } \\
\text { algorithm }\end{array}$ \\
\hline 1 & 2342 & 2198 & 2486 & 2599 & 2466 & 2732 & 147 & 155 & 138 \\
\hline 2 & 4300 & 4177 & 4423 & 3850 & 3737 & 3962 & 136 & 130 & 141 \\
\hline 3 & 5187 & 5225 & 5149 & 4495 & 4463 & 4526 & 222 & 210 & 234 \\
\hline 4 & 3938 & 4003 & 3872 & 3819 & 3679 & 3957 & 150 & 156 & 144 \\
\hline 5 & 3009 & 2916 & 3102 & 2157 & 2075 & 2239 & 178 & 185 & 170 \\
\hline 6 & 2694 & 2638 & 2749 & 3159 & 3077 & 3241 & 154 & 146 & 161 \\
\hline 7 & 3427 & 3481 & 3372 & 2629 & 2595 & 2663 & 131 & 138 & 124 \\
\hline
\end{tabular}

where $K$ is the correction coefficient of the inversion modulus of the surface course and $T$ is the expressed temperature.

4.2. Humidity Correction of Inversion Subgrade Modulus in Seasonal Frozen Area. Temperature in different seasons fluctuates greatly in the seasonal frozen area. Water in the subgrade turns into ice at the temperature below $0^{\circ} \mathrm{C}$, which changes the properties of subgrade structure materials as well as the position and volume of soil particles. In contrast, ice turns into water at the temperature above $0^{\circ} \mathrm{C}$, which causes the volume of soil particles to become smaller. Both factors will result in repeated frost heaving and thawing settlement deformation of pavement. Therefore, considering 


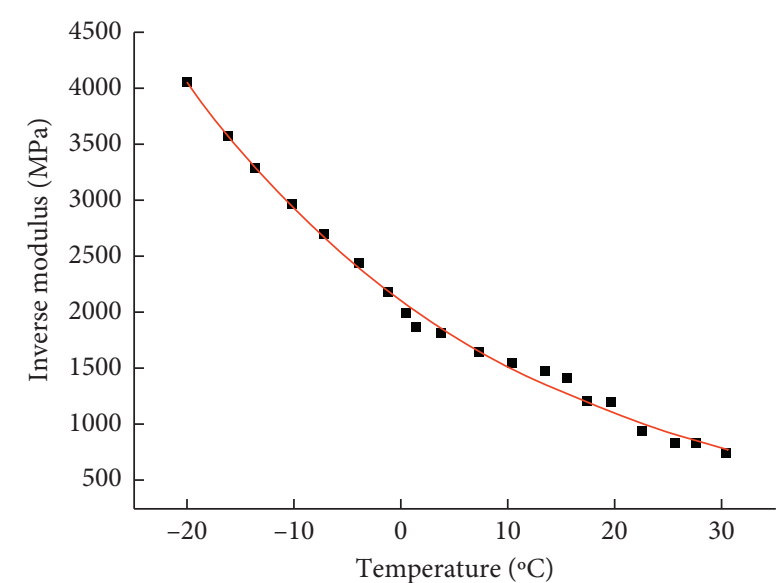

Figure 7: Correlation between inversion modulus of asphalt surface course and temperature.

TABLE 6: Correlating data of pavement temperature and inversion modulus of surface course.

\begin{tabular}{lc}
\hline Temperature $\left({ }^{\circ} \mathrm{C}\right)$ & Inverse modulus $(\mathrm{MPa})$ \\
\hline-20.1 & 4059 \\
-16.2 & 3585 \\
-13.6 & 3299 \\
-10.2 & 2964 \\
-7.2 & 2697 \\
-3.9 & 2441 \\
-1.2 & 2186 \\
0.5 & 2002 \\
1.4 & 1873 \\
3.7 & 1816 \\
7.3 & 1641 \\
10.4 & 1544 \\
13.5 & 1481 \\
15.5 & 1419 \\
17.4 & 1209 \\
19.6 & 1200 \\
22.6 & 939 \\
25.7 & 8299 \\
27.6 & 837 \\
30.5 & 743 \\
\hline
\end{tabular}

the influence of subgrade water content on subgrade modulus inversion is of great significance to evaluate the performance of the asphalt concrete pavement structure layer in the seasonal frozen area.

The following basic assumptions are made to calculate the humidity field:

(1) The asphalt concrete pavement is simplified as a multilayer elastic system, assuming that the layers are continuous and compact and the materials of each layer are homogeneous and isotropic

(2) The moisture transmission only occurs on the crosssection of the asphalt concrete pavement

(3) The moisture change of the subgrade is only related to the initial water content and groundwater without regard to the external moisture movement among the structures

(4) The moisture movement is carried out in the subgrade regardless of moisture migration in other structural layers

The humidity field of subgrade with the above assumption can be described by the following humidity field equation with phase transition:

$$
\frac{\partial w}{\partial t}=\frac{\partial}{\partial x}\left[D_{x}(w) \frac{\partial w}{\partial x}\right]+\frac{\partial}{\partial y}\left[D_{y}(w) \frac{\partial w}{\partial y}-K_{y}(w)\right] \quad t>0,
$$

where $w$ is the water content; $K(w)$ is the hydraulic conductivity; $D(w)$ is the diffusivity.

The final moisture content of any point in the seasonal frozen area is calculated as follows: suppose that the phase change interface reaches here at time $t_{0}$, and its water content is $w\left(t_{0}, x, y\right)$; when the phase change interface develops downward at time $t_{1}$, the final water content of this point is

$$
\begin{aligned}
w(x, y)= & w\left(t_{0}, x, y\right)+\left(t_{1}-t_{0}\right) \\
& \cdot\left[\left(D_{y}(w) \frac{\partial w}{\partial y}-K_{y}(w)\right)+D_{x}(w) \frac{\partial w}{\partial x}\right] .
\end{aligned}
$$

The hydraulic conductivity $K(w)$ is related to dry density $r_{d}$ and saturation $G$ as follows:

$$
K\left(w, r_{d}\right)=0.046\left(\frac{2.7-r_{d}}{1.44}\right)^{14.79} G^{9.69}
$$

The diffusivity $D(w)$ is related to dry density $r_{d}$ and saturation $G$ as follows:

$$
D\left(w, r_{d}\right)=10.28\left(\frac{2.7-r_{d}}{1.44}\right)^{11.44} G^{2.72}
$$

The correlation between subgrade modulus and humidity can be obtained by embedding the model into the inversion program. The calculation results are shown in Figure 8 and Table 7.

Figure 8 shows that the inversion modulus of subgrade decreases with the increase of water content, which is an exponential relationship. The correlation formula of inversion modulus of subgrade and water content is obtained from regression analysis as follows:

$$
E=215.97 e^{-0.049 w} \text {. }
$$

The correlation coefficient $R^{2}$ between them is equal to 0.9833 .

The humidity correction coefficient $K_{E}$ is defined as the ratio between the modulus $E$ of subgrade with water content $w$ and the modulus $E_{S}$ under the condition of optimal water content $w_{S}$. The optimal water content $w_{S}$ is $16 \%$, that is,

$$
K_{E}=\frac{E}{E_{s}}=\frac{215.97 e^{-0.049 w}}{215.97 e^{-0.049 w_{s}}}=e^{-0.049(w-16)},
$$




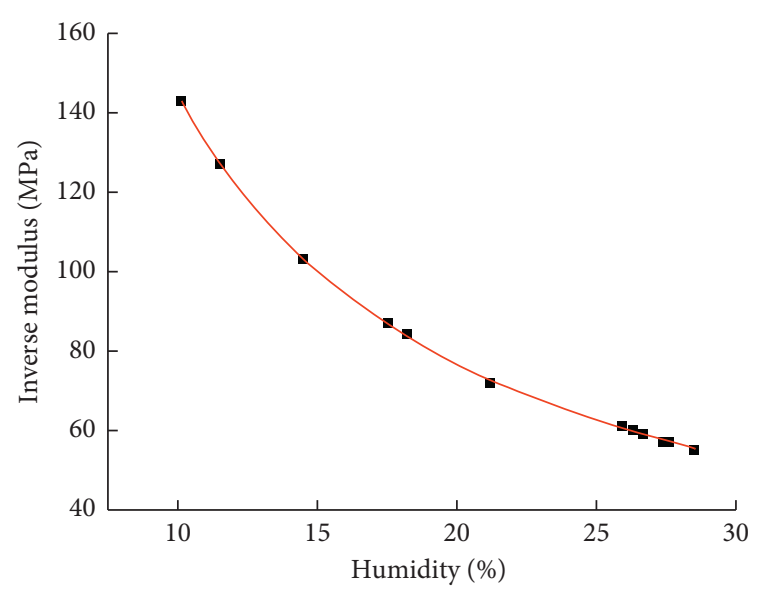

FIGURE 8: Correlation between inversion modulus of subgrade and humidity.

TABLE 7: Subgrade humidity and inversion modulus data.

\begin{tabular}{lc}
\hline Humidity (\%) & Inverse modulus $(\mathrm{MPa})$ \\
\hline 26.70 & 59 \\
27.60 & 57 \\
27.40 & 57 \\
26.30 & 60 \\
28.50 & 55 \\
21.20 & 72 \\
17.50 & 87 \\
18.20 & 84 \\
11.50 & 127 \\
10.10 & 143 \\
14.50 & 103 \\
25.90 & 61 \\
\hline
\end{tabular}

where $K_{E}$ is the correction coefficient of inversion modulus of subgrade and $w$ is the water content.

\section{Conclusion}

In this paper, in view of the existing problems in the inversion of the asphalt concrete pavement structure layer modulus, the support vector machine model is introduced into the asphalt concrete pavement modulus inverse analysis to study the influences of the temperature and humidity in the seasonal frozen area on the inversion modulus. The research conclusions are as follows:

(1) After inversion by using the support vector machine model optimized by the firefly algorithm, the average of relative error absolute value of the pavement structural layer modulus is within $2 \%$ and the maximum value is within $4 \%$, which meets the accuracy requirements.

(2) Considering the influence of temperature and humidity on the inversion modulus, the inversion modulus of pavement surface layer will decrease with the increase of temperature, and the inversion modulus of subgrade will decrease with the increase of water content.
(3) According to the inversion results, the modified formulas for the inversion modulus of the semirigid base pavement surface layer and subgrade varying with temperature and humidity are established: $K=e^{-0.033(T-20)}$ and $K_{E}=e^{-0.049(w-16)}$.

\section{Data Availability}

The data used to support the findings of this study are not available.

\section{Conflicts of Interest}

The authors declare that they have no conflicts of interest.

\section{Acknowledgments}

The authors acknowledge the support received from the National Key Research and Development Plan (Grant no. 2018YFB1600200), the National Natural Science Foundation of China (Grant nos. 51878624 and 51878622), the Major Scientific and Technological Special Project in Henan (Grant no. 181100310400), the Program for Innovative Research Team (in Science and Technology) at the University of Henan Province (Grant no. 18IRTSTHN007), the Program for Science and Technology Innovation Talents in Universities of Henan Province (Grant no. 19HASTIT041), and the Key Research Projects of Higher Education in Henan Province (Grant no. 18A580001).

\section{References}

[1] C. Jing, J. Zhang, and B. Song, "An innovative evaluation method for performance of in-service asphalt pavement with semi-rigid base," Construction and Building Materials, vol. 235, 2020.

[2] A. Meng, "Feasibility of freeze-thaw damage analysis for asphalt mixtures through dynamic nondestructive testing," Construction and Building Materials, vol. 233, 2020.

[3] G. L. Golewski, "A novel specific requirements for materials used in reinforced concrete composites subjected to dynamic loads," Composite Structures, vol. 223, no. 223, 2019.

[4] G. L. Golewski, "New principles for implementation and operation of foundations for machines: a review of recent advances," Structurat Engineering and Mechanics, vol. 71, pp. 317-327, 2019.

[5] M. Li, H. Wang, G. Xu, and P. Xie, "Finite element modeling and parametric analysis of viscoelastic and nonlinear pavement responses under dynamic FWD loading," Construction and Building Materials, vol. 141, pp. 23-35, 2017.

[6] H. Scrivner and W. F. Mcfarland, "A systems approach to the flexible pavement design problem,” pp. 20-70, 1968, Research report.

[7] N. Sivaneswaran, S. L. Kramer, and J. P. Mahoney, "Advanced backcalculation using a nonlinear least squares optimization technique," Journal of the Transportation Research Board, vol. 1239, 1991.

[8] F. Wang and R. L. Lytton, "System identification method for backcalculating pavement layer properties," Journal of the Transportation Research Board, vol. 1384, pp. 1-7, 1993. 
[9] T. Scullion, J. Uzan, and M. Paredes, "Modulus: a microcomputer-based backcalculation system," Transportation Research Record, vol. 1260, pp. 180-191, 1990.

[10] R. W. Meier, D. R. Alexander, and R. B. Freeman, "Using artificial neural networks as a forward approach to backcalculation," Transportation Research Record: Journal of the Transportation Research Board, vol. 1570, no. 1, pp. 126-133, 1997.

[11] T. F. Fwa, C. Y. Tan, and W. T. Chan, "Backcalculation analysis of pavement-layer moduli using genetic algorithms," Transportation Research Record: Journal of the Transportation Research Board, vol. 1570, no. 1, pp. 134-142, 1997.

[12] H. Wang, M. Li, P. Szary, and X. Hu, "Structural assessment of asphalt pavement condition using back calculated modulus and field data," Construction and Building Materials, vol. 211, pp. 943-951, 2019.

[13] L. You, K. Yan, and N. Liu, "Assessing artificial neural network performance for predicting interlayer conditions and layer modulus of multi-layered flexible pavement," Frontiers of Structural and Civil Engineering, vol. 12, 2020.

[14] M. Li and H. Wang, "Development of ANN-GA program for back calculation of pavement moduli under FWD testing with viscoelastic and nonlinear parameters," International Journal of Pavement Engineering, vol. 20, no. 4, pp. 490-498, 2019.

[15] J. C. Edwin and J. G. Anthony, "Effect of freezing and thawing on the permeability and structure of soils[J]," Engineering Geology, vol. 13, no. 1-4, pp. 73-92, 1979.

[16] E. Simonsen, V. C. Janoo, and U. Isacsson, "Resilient properties of unbound road materials during seasonal frost conditions," Journal of Cold Regions Engineering, vol. 16, no. 1, pp. 28-50, 2002.

[17] X. Mao, Z. Hou, and W. Wang, "Experimental study on the resilience modulus of remolded soil based on water content and freeze-thaw cycles," Chinese Journal of Rock Mechanics \& Engineering, vol. 28, pp. 3585-3590, 2009.

[18] W. Chen, "Study on modulus of resilience and humidity of subgrade seasonal frozen region," Master Degree Thesis, Wuhan Polytechnic University, Hubei, China, 2012.

[19] M. Wang, "Research on moisture and temperature monitoring and intensity change of subgrade in seasonal frozen region," Master Degree Thesis, Northeast Forestry University, Harbin, China, 2014.

[20] M. Williams, Structural Dynamics, Taylor \& Francis, Milton Park, UK, 2016.

[21] I. Babuška and T. Strouboulis, The Finite Element Method and its Reliability, Clarendon Press, Oxford, UK, 2001.

[22] X. Qiu, Research on modulus parameter inversion and correction method of semi-rigid base asphalt pavement based on FWD, PhD Thesis, Tongji University, Shanghai, China, 2009.

[23] V. Vapnik, "Statistical Learning Theory," Encyclopedia of the Sciences of Learning, vol. 41, no. 4, pp. 3185-3185, 1998.

[24] C. Cortes and V. Vapnik, "Support-vector networks," Machine Learning, vol. 20, no. 3, pp. 273-297, 1995.

[25] V. Cherkassky and Y. Ma, "Practical selection of SVM parameters and noise estimation for SVM regression," Neural Networks, vol. 17, no. 1, pp. 113-126, 2004.

[26] K. Ito and R. Nakano, "Optimizing support vector regression hyperparameters based on cross-validation," in Proceedings of the 2003 International Joint Conference, Portland, OR, USA, 2003.

[27] K. N. Krishnanand and D. Ghose, "Detection of multiple source locations using A glowworm mmetaphor with applications to collective robotics," in Proceedings 2005 IEEE Swarm Intelligence Symposium, Pasadena, CA, USA, 2005.
[28] F. Budiman, "SVM-RBF parameters testing optimization using cross validation and grid search to improve multiclass classification," Scientific Visualization, vol. 11, no. 1, pp. 80-90, 2019.

[29] R. Zhang, "Study on temperature field distribution law of pavement structure in seasonally frozen regions considering extreme climate," Master Degree Thesis, Harbin Institute of Technology, Harbin, China, 2012. 